\section{Peripheral and posterior pole retinal lesions in association with high myopia: a cross-sectional community-based study in Hong Kong}

TYY Lai, DSP Fan, WWK Lai and DSC Lam

\begin{abstract}
Purpose To evaluate the prevalence and factors associated with posterior pole and peripheral retinal lesions in Chinese subjects with high myopia.

Methods Three hundred and thirty-seven asymptomatic adults with high myopia of refractive error $\leqslant-6 \mathrm{D}$ were examined in a cross-sectional community-based study. All subjects underwent cycloplegic refraction, ultrasound biometry and dilated fundal examination. Statistical analysis was performed to assess factors associated with the presence of posterior pole and peripheral retinal lesions.
\end{abstract}

Results The mean age of the 337 subjects was 36.0 years and the mean spherical equivalent refractive error was $-10.2 \mathrm{D}$. Thirty-eight eyes (11.3\%, 95\% CI $=\mathbf{8 . 1 - 1 5 . 2 \% )}$ were found to have one or more posterior pole lesions and subjects with posterior pole lesion had significantly older age, longer axial length and higher degree of myopia (all $P<0.001$ ) compared with subjects without posterior pole lesion. After controlling for axial length, both the severity of refractive error and older age were significantly associated with the presence of posterior pole lesion (both $\boldsymbol{P}<\mathbf{0 . 0 0 1}$ ). For peripheral retinal lesions, 189 eyes $(56.1 \%, 95 \%$ $\mathrm{CI}=50.6-61.5 \%)$ were found to have one or more peripheral retinal lesions. The presence of peripheral retinal lesion was associated with younger age and higher degree of refractive error $(P=0.046$ and 0.002 , respectively).

Conclusion Posterior pole and peripheral retinal degenerative lesions were found in a considerable proportion of subjects with high myopia. As some of these retinal lesions might predispose to visual impairment, highly myopic individuals should be educated on the symptoms of various eye conditions and seek care immediately if symptoms arise.

Eye (2008) 22, 209-213; doi:10.1038/sj.eye.6702573; published online 1 September 2006

Keywords: prevalence; peripheral retinal degeneration; macula; posterior pole; high myopia

\section{Introduction}

High myopia or pathological myopia is associated with globe elongation and a refractive error of at least -6 dioptres (D) and/or an axial length of $>25.5 \mathrm{~mm} .{ }^{1-3}$ The prevalence of high myopia varies considerably in different population and ranges from $<1 \%$ in Afro-American population to more than $10 \%$ in Asian populations. ${ }^{1,2}$ Individuals with pathologic myopia have increased risks of retinal complications such as lattice degeneration, retinal detachment, choroidal neovascularisation, and macular haemorrhage. ${ }^{4-6}$ As some of the retinal complications are potentially sight-threatening, understanding the risk factors for the development of these complications in high myopia will be beneficial to identify these high-risk subjects.

Several studies have demonstrated increased prevalence of peripheral retinal degenerations in association with high myopia and increased
Department of Ophthalmology and Visual Sciences, The Chinese University of Hong Kong, Hong Kong Eye Hospital, Kowloon, Hong Kong

Correspondence:

DSC Lam, Department of Ophthalmology and

Visual Sciences,

The Chinese University of Hong Kong, 3/F, Hong Kong Eye Hospital,

147K Argyle Street, Kowloon, Hong Kong Tel: + 85227623157 Fax: +85227159490 .

E-mail: dennislam_pub@ cuhk.edu.hk

Received: 9 February 2006 Accepted in revised form: 28 July 2006

Published online:

1 September 2006

Financial support: Supported in part by Action For Vision (AFV) Eye Foundation, Hong Kong

Financial interest: Ni 
axial length. ${ }^{4-12}$ Although some studies have found association between increased axial length or the severity of myopia with peripheral retinal degenerations, some of the results were conflicting and the correlation between axial length and peripheral retinal changes is not clearly established. ${ }^{5,6}$ In studies by Pierro et $a l^{5}$ and Gozum et $a l^{11}$ it was found that longer axial length was associated with increased prevalence of peripheral retinal degenerations including lattice degeneration, paving stone degeneration, and white without pressure. However, no correlation was observed between high myopia and peripheral retinal pigment, retinal tears and retinal detachment. ${ }^{5}$ Celorio et al also reported that the peak prevalence of lattice degeneration was in eyes with axial lengths of $26.0-26.9 \mathrm{~mm}$, followed by an inverse relationship between the prevalence of lattice degeneration and eyes with axial lengths $>27.0 \mathrm{~mm}^{4}{ }^{4}$ One of the reasons for the conflicting results in these studies might be due to the intrinsic limitations with retrospective data collection and clinic-based subjects recruitment. Moreover, since the prevalence of retinal degenerative findings might be related to age, differences in the age spectrum among these studies might also influence the findings.

Besides the increased prevalence of peripheral retinal degeneration in high myopia, studies have also suggested an increase risk of posterior pole chorioretinal abnormalities in subjects with high myopia. ${ }^{11,12}$ However, the prevalence of posterior pole abnormalities among highly myopic eyes have not be assessed adequately in the literature due to lack of communitybased cross-sectional or case-control series. ${ }^{12}$ Therefore, we performed a community-based study of asymptomatic highly myopic subjects in order to determine the prevalence of posterior pole and peripheral chorioretinal lesions in high myopes among Hong Kong Chinese population. We also aimed at investigating the relationship between axial length, refractive error, and age with the presence of posterior pole and peripheral retinal abnormalities.

\section{Subjects and methods}

This was a cross-sectional population-based study performed in Hong Kong in October 1996 and August 2004. All subjects were recruited from the community after advertising through the media. The inclusion criteria were as follow: ethnic Chinese; spherical equivalent refractive error of $\leqslant-6.0 \mathrm{D}$ in at least one eye; and age 18 years or older. Subjects with a history of prematurity, previous retinal diseases including retinal detachment, or eyes with media opacity such as corneal scar or cataract obscuring detailed examination were excluded from the study. Some of the findings among the subjects have been reported previously. ${ }^{13}$ The protocol was approved by the institutional review board at the Chinese University of Hong Kong and informed consent was obtained from each of the invited participants.

All participants received a comprehensive ocular examination, which included intraocular pressure (IOP) measurement by noncontact tonometer (Rechert XPERT NCT Plus, New York, USA), slit-lamp anterior segment examination, noncycloplegic, and cycloplegic autorefraction (Topcon KR 7100, Tokyo, Japan), and A-Scan ultrasound biometry (Storz Compuscan LT, St Louis, USA). Dilated retinal examination was performed by vitreoretinal fellows or specialists with indirect ophthalmoscope and scleral indentation together with slit-lamp biomicroscopy using contact lens were performed if required.

Ocular measurements including refraction, spherical equivalent refractive error, IOP, axial length, and findings of fundus examinations including posterior pole and peripheral retinal lesions were recorded. Statistical analysis was performed using SPSS v11.5. Two-tailed $t$-test was performed to compare the refractive error, biometric measurements and age of subjects with or without retinal lesions. Univariate analysis of variance was performed to determine factors significantly associated with the presence of posterior pole and peripheral retinal lesion. The mean age and spherical equivalent refractive error were also calculated for eyes with or without the posterior pole and peripheral retinal lesions after adjusted for axial length measurements by multivariate analysis.

\section{Results}

\section{Subjects demographics}

A total of 337 subjects with a refractive error of $\leqslant-6.0 \mathrm{D}$ measured by cycloplegic refraction fulfilled the inclusion criteria and were analysed. Two hundred and eighty-two $(83.7 \%)$ subjects had bilateral high myopia while 55 $(26.4 \%)$ had unilateral high myopia. The data of the right eyes of subjects with bilateral high myopia were used for further analysis. For unilateral cases, only the data of the highly myopic eyes were used. The mean \pm standard deviation (SD) age of the subjects was $36.0 \pm 12.2$ years (range: $18-73$ years). There were 238 females $(70.6 \%)$ and 99 males $(29.4 \%)$. The mean \pm SD spherical equivalent refractive error was $-10.2 \pm 4.0 \mathrm{D}$ (range: -6.0 to $-26.5 \mathrm{D}$ ) and the mean \pm SD astigmatism was $1.30 \pm 1.11 \mathrm{D}$ (range: $0.0-5.5 \mathrm{D}$ ). The mean $\pm \mathrm{SD}$ axial length of the eyes was $26.84 \pm 1.57 \mathrm{~mm}$ (range: $23.18-33.57 \mathrm{~mm}$ ). There was a strong correlation between the spherical equivalent refractive error and axial length $(r=-0.704, P<0.001)$. 


\section{Presence of posterior pole chorioretinal lesions}

Of the 337 eyes analysed, 38 (11.3\%) eyes were found to have one or more posterior pole lesions including chorioretinal atrophy, Fuch's spot, lacquer crack, and posterior staphyloma (Table 1). Univariate analysis showed eyes with the presence of posterior pole lesion had significantly older age (45.0 vs 34.8 years), longer axial length (28.84 vs $26.59 \mathrm{~mm}$ ), and higher degree of mean spherical equivalent refractive error $(-16.8$ vs $-9.4 \mathrm{D})(P<0.001$ for all three variables). The mean $\pm \mathrm{SD}$ spherical equivalent refractive error for eyes with and without peripheral retinal degeneration was $-16.8 \pm 5.5$ and $-9.4 \pm 2.9 \mathrm{D}$, respectively; and the mean $\pm \mathrm{SD}$ axial length of eyes with or without posterior pole lesions was $28.84 \pm 2.25$ and $26.59 \pm 1.27 \mathrm{~mm}$, respectively. Eyes with axial length of $\geqslant 29 \mathrm{~mm}$ were more likely to have posterior pole chorioretinal lesion including chorioretinal atrophy and lacquer cracks compared with eyes with axial length of $<29 \mathrm{~mm}$ ( $\chi^{2}$ test, $P<0.001$ ). After controlling for axial length findings, multivariate analysis showed both refractive error and older age were significantly related to the presence of posterior pole lesion $(P<0.001$ for both analysis). The adjusted mean age and spherical equivalent refractive error of subjects with posterior pole lesion were 43.6 years and $-13.9 \mathrm{D}$, respectively. This compared to the adjusted mean age of 34.8 years and spherical equivalent refractive error of $-9.4 \mathrm{D}$ for subjects without posterior pole lesion.

Table 1 Number and proportion of eyes with posterior pole chorioretinal lesions

\begin{tabular}{lcc}
\hline $\begin{array}{l}\text { Type of peripheral retinal } \\
\text { lesion }\end{array}$ & $\begin{array}{c}\text { Number of } \\
\text { eyes }\end{array}$ & $\begin{array}{c}\text { Proportion of eyes } \\
(95 \% \mathrm{CI})\end{array}$ \\
\hline Posterior staphyloma & 26 & $7.7(5.1-11.0 \%)$ \\
Chorioretinal atrophy & 9 & $2.7(1.2-5.0 \%)$ \\
Lacquer crack & 6 & $1.8(0.6-3.8 \%)$ \\
Fuch's spot & 1 & $0.3(0.0-1.6 \%)$ \\
Any posterior pole lesion & 38 & $11.3(8.1-15.2 \%)$ \\
\hline
\end{tabular}

$95 \% \mathrm{CI}=95 \%$ confidence interval.

Table 2 Number and proportion of eyes with peripheral retinal lesions

\begin{tabular}{lcc}
\hline Type of peripheral retinal lesion & $\begin{array}{c}\text { Number } \\
\text { of eyes }\end{array}$ & $\begin{array}{c}\text { Proportion of } \\
\text { eyes }(95 \% \mathrm{CI})\end{array}$ \\
\hline $\begin{array}{l}\text { Pigmentary peripheral } \\
\text { retinal degeneration }\end{array}$ & 127 & $37.7(32.5-43.1 \%)$ \\
$\begin{array}{l}\text { White without pressure } \\
\text { Lattice degeneration }\end{array}$ & 71 & $21.1(16.8-25.8 \%)$ \\
$\begin{array}{l}\text { Peripheral retinal hole } \\
\text { or break }\end{array}$ & 46 & $13.6(10.2-17.8 \%)$ \\
Any peripheral lesion & 21 & $6.2(3.9-9.4 \%)$ \\
\hline
\end{tabular}

$95 \% \mathrm{CI}=95 \%$ confidence interval.

\section{Presence of peripheral retinal lesions}

There were 189 (56.1\%) eyes found to have one or more peripheral retinal degenerative lesion including retinal hole/break, pigmentary degeneration, lattice degeneration and white without pressure (Table 2). The mean \pm SD spherical equivalent refractive error for eyes with and without peripheral retinal lesion was $-11.0 \pm 4.5$ and $-9.3 \pm 3.1 \mathrm{D}$, respectively. Eyes with the presence of peripheral retinal degenerative lesion had significantly higher degree of myopia compared with eyes without peripheral retinal lesion $(P<0.001)$. For subjects with unilateral high myopia, there was a higher prevalence of peripheral retinal lesions in the more myopic eyes but the difference was not statistically significant $(P=0.45)$. The mean axial length of eyes with peripheral retinal lesion was significantly higher compared with eyes without peripheral retinal lesion, with the mean \pm SD of $27.04 \pm 1.69$ and $26.59 \pm 1.37 \mathrm{~mm}$, respectively $(P=0.009)$. Eyes with axial length of $\geqslant 29 \mathrm{~mm}$ were more likely to have peripheral retinal lesion compared with those with axial length of $<29 \mathrm{~mm}(P=0.05)$. After controlling for axial length measurements, multivariate analysis showed both younger age and refractive errors were significantly related to the presence of peripheral retinal lesion (one-way ANOVA, $P=0.046$ and 0.002 , respectively). The adjusted mean age and spherical equivalent refractive error of subjects with peripheral lesion were 34.7 years and $-10.6 \mathrm{D}$, respectively, compared to 37.4 years and $-9.6 \mathrm{D}$ for subjects without peripheral retinal lesions.

There was also significant association between the presence of posterior pole and peripheral retinal lesions $(P=0.020)$. However, after adjusted for axial length measurements or spherical equivalent refractive error, the association was no longer statistically significant $(P=0.12$ and 0.74 , respectively).

\section{Discussion}

In this cross-sectional study of asymptomatic community-based individuals, we found that more than $50 \%$ of subjects had one or more peripheral retinal lesion and $11 \%$ of subjects had posterior pole chorioretinal lesion. The commonest peripheral retinal lesion noted was pigmentary degeneration followed by white without pressure. Peripheral retinal degenerative lesions such as lattice degeneration and retinal holes or breaks, which are considered important risk factors for retinal detachment, were found in 13.6 and $6.2 \%$ of eyes, respectively. Previous studies on the association of retinal lesion and high myopia have shown large variations in findings due to differences in subject recruitment method 
and most subjects were recruited from clinic-based practices. $^{4-12}$ Moreover, the prevalence of myopia is dependent on the subjects' ethnic background and therefore results from other studies might not be generalisable to various populations. The majority of population in Hong Kong is ethnic Chinese and the prevalence of high myopia among this urban Chinese population is one of the highest in the world. ${ }^{14}$ By studying a community-based sample of subjects, we aimed to estimate the prevalence of retinal abnormalities in association with high myopia as well as determining the association between retinal lesions with axial length and high myopia. This will minimise the bias associated with some of the previous clinic-based studies. Despite our study having included a homogeneous population of Hong Kong Chinese adults, our study sample might still have some bias due to self-selection of the population. Another potential source of bias might be the exclusion of individuals with previous history of retinal diseases like those who had retinal detachment and the prevalence of retinal pathology in our sample might be an underestimation.

Our study evaluated the association of posterior pole chorioretinal lesions with axial length and spherical equivalent refractive error. Eyes with chorioretinal lesions at the posterior pole had significantly longer axial length and higher magnitude of myopia compared with those without posterior pole lesions. Around 10\% of eyes with axial length of $\geqslant 29 \mathrm{~mm}$ had lacquer crack on fundus examination, compared with only $1 \%$ of eyes with axial length of $<29 \mathrm{~mm}$. Older age and higher magnitude of refractive error were also found to be independently associated with the presence of posterior pole chorioretinal lesions after adjustment of axial length findings. The $11 \%$ prevalence of posterior pole lesion observed in our study was less compared with the rate of around $25 \%$ in subjects with myopia of $<-5 \mathrm{D}$ in Blue Mountain Eye Study. ${ }^{15}$ The main reason is likely to be due to the age differences between the two studies as only subjects aged 49 years or older were included in the Blue Mountain Eye Study, whereas we included subjects aged 18 years or older. ${ }^{15}$ As posterior staphyloma and lacquer cracks are known to be associated with the development of macular hole retinal detachment and choroidal neovascularisation, respectively, ${ }^{16,17}$ it is therefore important to inform older patients with these posterior pole lesions to seek ophthalmic care as soon as new symptoms arise.

Our results also demonstrated there were strong associations between axial length and various peripheral retinal lesion and posterior pole chorioretinal lesions. $54.5 \%$ of eyes with axial length of $<29 \mathrm{~mm}$ compared to $74.1 \%$ of eyes with axial length of $\geqslant 29 \mathrm{~mm}$ were found to have peripheral retinal lesions. The presence of retinal break was found in $5.6 \%$ of eyes with axial length of
$<29 \mathrm{~mm}$, compared to $11.1 \%$ of eyes with axial length of $\geqslant 29 \mathrm{~mm}$. This was consistent with previous study by Pierro $e t a l^{5}$ in which eyes with white without pressure, paving stone degeneration and lattice degeneration had significantly greater axial length. The prevalence of lattice degeneration in highly myopic Chinese as observed in our study was $13.6 \%$, which was slightly higher or comparable with those reported in other ethnic groups., ${ }^{46}$ Moreover, after adjusted analysis for axial length, our results also demonstrated that higher magnitude of spherical equivalent refractive error has an independent association with the presence of peripheral retinal lesion. As myopia and lattice degeneration were demonstrated to be important risk factors for retinal detachment, $9,17,18$ close follow-up of highly myopic patients with lattice degeneration in these patients might be warranted.

One of the reasons for the variation in results on the association of retinal lesions with refractive error and axial length observed in previous studies might be due to the influence of age in various studies. Our results showed that subjects with posterior pole lesions had significantly older age compared with those without posterior pole lesion, while subjects with peripheral retinal lesion had a younger age than those without peripheral lesion. The findings suggested that in high myopia, increasing age might not be a risk factor for the development of peripheral retinal lesions but might be a risk factor for macular lesions. These findings were consistent with the findings reported by Gozum et al, ${ }^{11}$ in which posterior pole lesions including chorioretinal atrophy, Fuchs' spot and posterior staphyloma were associated with older age, whereas peripheral retinal lesion like white-without-pressure was correlated with younger age. This was also in line with the findings from the Blue Mountain Eye Study, in which an age-related trend was observed for the prevalence of myopic macular lesions including posterior staphyloma, lacquer cracks, Fuchs' spot and chorioretinal atrophy. ${ }^{15}$ However, our results differed from those reported by Karlin and Curtin $^{8}$ in which increased age was significantly associated with the presence of pigmentary and paving stone degenerations. The main reason might be due to clinic-based selection in the study by Karlin and Curtin versus community-based selection in our study.

In summary, a substantial proportion of asymptomatic highly myopic subjects in this urban Chinese community-based study were found to have peripheral retinal degenerative as well as posterior pole chorioretinal lesions. As previous studies in highly myopic eyes have demonstrated these degenerative lesions might be associated with serious vision threatening complications, highly myopic patients should be educated about the symptoms of retinal 
complications such as retinal detachment and choroidal neovascularisation, and advised to seek ophthalmic care promptly should such symptoms arise.

\section{References}

1 Sperduto RD, Seigel D, Roberts J, Rowland M. Prevalence of myopia in the United States. Arch Ophthalmol. 1983; 101: 405-407.

2 Wu HM, Seet B, Yap EP, Saw SM, Lim TH, Chia KS. Does education explain ethnic differences in myopia prevalence? A population-based study of young adult males in Singapore. Optom Vis Sci 2001; 78: 234-239.

3 Grossniklaus HE, Green WR. Pathological Findings in Pathologic Myopia. Retina 1992; 12: 127-133.

4 Celorio JM, Pruett RC. Prevalence of lattice degeneration and its relation to axial length in severe myopia. Am J Ophthalmol 1991; 111: 20-23.

5 Pierro L, Camesasca FI, Mischi M, Brancato R. Peripheral retinal changes and axial myopia. Retina 1992; 12: 12-17.

6 Hyams SW, Neumann E. Peripheral retinal in myopia. With particular reference to retina breaks. Br J Ophthalmol 1969; 53: 300-306.

7 Curtin BJ, Karlin DB. Axial length measurements and fundus changes of the myopic eye. I. The posterior fundus. Trans Am Ophthalmol Soc 1970; 68: 312-334.

8 Karlin DB, Curtin BJ. Peripheral chorioretinal lesions and axial length of the myopic eye. Am J Ophthalmol 1976; 81: 625-635.
9 The Eye Disease Case-Control Study Group. Risk factors for idiopathic rhegmatogenous retinal detachment. Am J Epidemiol 1993; 137: 749-757.

10 Yura T. The relationship between the types of axial elongation and the prevalence of lattice degeneration of the retina. Acta Ophthalmol Scand 1998; 76: 90-95.

11 Gozum N, Cakir M, Gucukoglu A, Sezen F. Relationship between retinal lesions and axial length, age and sex in high myopia. Eur J Ophthalmol 1997; 7(3): 277-282.

12 Saw SM, Gazzard G, Shih-Yen EC, Chua WH. Myopia and associated pathologic complications. Ophthalmic Physiol Opt 2005; 25: 381-391.

13 Lam DS, Fan DS, Chan WM, Tam BS, Kwok AK, Leung AT et al. Prevalence and characteristics of peripheral retinal degeneration in Chinese adults with high myopia: a crosssectional prevalence survey. Optom Vis Sci 2005; 82: 235-238.

14 Fan DS, Lam DS, Lam RF et al. Prevalence, incidence, and progression of myopia of school children in Hong Kong. Invest Ophthalmol Vis Sci 2004; 45: 1071-1075.

15 Vongphanit J, Mitchell P, Wang JJ. Prevalence and progression of myopic retinopathy in an older population. Ophthalmology 2002; 109: 704-711.

16 Lam DS, Tam BS, Chan W, Leung AT. Retinal breaks. Ophthalmology 1999; 106: 859-860.

17 Burton TC. The influence of refractive error and lattice degeneration on the incidence of retinal detachment. Trans Am Ophthalmol Soc 1989; 87: 143-155.

18 Avila MP, Weiter JJ, Jalkh AE, Trempe CL, Pruett RC, Schepens CL. Natural history of choroidal neovascularization in degenerative myopia. Ophthalmology 1984; 91: 1573-1581. 